\title{
CLASSES SUCESSIONAIS E SÍNDROMES DE DISPERSÃO DE ESPÉCIES ARBÓREAS E ARBUSTIVAS EXISTENTES EM VEGETAÇÃO CILIAR REMANESCENTE (CONCHAL, SP), COMO SUBSÍDIO PARA AVALIAR O POTENCIAL DO FRAGMENTO COMO FONTE DE PROPÁGULOS PARA ENRIQUECIMENTO DE ÁREAS REVEGETADAS NO RIO MOGI-GUAÇU, SP'
}

\author{
Clarissa de Aquino² e Luiz Mauro Barbosa ${ }^{3}$
}

\begin{abstract}
RESUMO - Este estudo foi realizado em fragmento de vegetação ciliar remanescente, localizado à margem direita do rio Mogi-Guaçu, Município de Conchal, SP, tendo como objetivo a avaliação do potencial desse fragmento como fonte de propágulos para projetos de enriquecimento em áreas ciliares implantadas na região de Mogi-Guaçu, SP. Para tanto, foi realizada a caracterização sucessional e da síndrome de dispersão das espécies arbóreas e arbustivas. No local, foram registradas 99 espécies arbóreas e arbustivas, pertencentes a 38 famílias. As famílias com maior número de espécies foram Euphorbiaceae, Fabaceae, Myrtaceae e Rubiaceae. Quanto aos grupos sucessionais, foi verificado equilíbrio na quantidade de espécies secundárias tardias $(28,3 \%)$ e daquelas típicas de sub-bosque $(23,2 \%)$. A síndrome de dispersão predominante entre as espécies arbóreas e arbustivas foi a zoocoria, identificada em $64,6 \%$ delas, seguida pela anemocoria, que representou $20,2 \%$ dos casos. Os resultados gerais apontaram o bom estado de conservação desse fragmento e a viabilidade de sua utilização como fonte de propágulos para ações de revegetação em áreas ciliares da região.
\end{abstract}

Palavras-chave: Área degradada, sementes e manejo.

SUCCESSIONAL GROUPS AND DISPERSAL SYNDROMES OF TREE AND SHRUB SPECIES AT THE RIPARIAN FOREST FRAGMENT IN CONCHAL, SP, BRAZIL TO EVALUATE ITS POTENTIAL AS A SOURCE OF SEEDS TO THE ENRICHMENT OF REVEGETATED RIPARIAN AREAS ALONG THE MOGIGUAÇU RIVER, SP

\begin{abstract}
This work was conducted in a reminiscent Riparian forest fragment at the Mogi-Guaçu River, in Conchal, SP, Brazil, to evaluate the potential of this fragment as a seed supplier to other projects regarding the preservation and enrichment of this type offorest in the region. Thus, the tree and scrub species sampled in floristic surveys were classified according to their dispersal syndromes and successional groups, with 38 families and 99 species being recorded in the area. The families with the most species were Euphorbiaceae, Fabaceae, Myrtaceae, and Rubiaceae. A balance was observed between late secondary species (28.3\%) and understory species (23.2\%). Zoochory was the most frequently observed syndrome (64.6\%), followed by anemochory (20.2\%). These results show the good conservation of the fragment studied, and confirm the viability of using it as a source of seeds in Riparian forest revegetation projects in the Mogi-Guaçu region.
\end{abstract}

Keywords: degraded area, seeds, management.

\footnotetext{
${ }^{1}$ Recebido em 28.05.2007 e aceito para publicação em 06.03.2009.

${ }^{2}$ Programa de Pós-Graduação em Biologia Vegetal da Universidade Estadual Paulista Júlio de Mesquita Filho (UNESP). E-mail: <clarissa.aquino@itelefonica.com.br>.

${ }^{3}$ Secretaria do Meio Ambiente, Instituto de Botânica de São Paulo-SP. E-mail: <lmbecol@terra.com.br>.
} 


\section{INTRODUÇÃO}

Formações florestais ribeirinhas ocorrem ao longo de cursos d'água e no entorno de nascentes, tendo características vegetacionais definidas em função da heterogeneidade ambiental, que é gerada pela interação de fatores físicos, climáticos e biológicos (CATHARINO, 1989; MANTOVANI et al., 1989; LOBO e JOLY, 1995; RODRIGUES, 2000). No Estado de São Paulo, tais formações, normalmente caracterizadas como matas ciliares, são compostas tanto por espécies exclusivas quanto por espécies de formações florestais do entorno, existindo baixa similaridade entre remanescentes. Fato também atribuído à intensa ação antrópica, que resultou em fragmentação e degradação desses ambientes (GIBBS eLEITÃOFILHO, 1978; BARBOSA, 1989; RODRIGUES e LEITÃO-FILHO, 2000).

A necessidade de proteção de sistemas hídricos e de sua biodiversidade, aliada à atual condição de fragmentação dos ambientes florestais, tem impulsionado a realização de trabalhos que visam à redução de impactos sobre remanescentes, além do desenvolvimento de medidas de políticas públicas e de modelos de recuperação de áreas degradadas que, normalmente, se dão através de plantios de revegetação com espécies arbóreas nativas (BARBOSA, 1989; RODRIGUES e LEITÃOFILHO, 2000; BARBOSA etal., 2003; BARBOSA, 2006).

Grande parte das práticas voltadas para recuperação de áreas degradadas (RAD) está centrada em conceitos de dinâmica, que se assemelham para as diversas formações florestais (PICKETT et al., 1987; DENSLOW, 1996; GRIME, 1998; METACALFE e TURNER, 1998). Com isso, a base para as ações de RAD vem da escolha adequada de espécies de plantio, baseada na autoecologia e comportamento silvicultural delas, associada a informações sobre: (a) padrões de produção de sementes e de recrutamento das espécies (BAWA e KRUGMAN, 1986; BARBOSA et al., 1992), (b) probabilidade de estabelecimento e velocidade de desenvolvimento das espécies num ambiente sucessional (BARBOSA et al., 1992; BENÍTEZ-MALVIDOeMARTINEZ-RAMOS, 2002) e (c) histórico de perturbação aliado às condições do entorno da área a ser recuperada ou manejada (KAGEYAMA e GANDARA, 2000).

Propostas recentes de RAD já incorporam, como prática de enriquecimento, a transferência de propágulos provenientes do banco e da chuva de sementes de fragmentos remanescentes, para áreas revegetadas que apresentem baixa resiliência e baixo input de sementes (AQUINO, 2006; BARBOSA, 2006). Essas ações estão pautadas no conceito de que a dinâmica da regeneração em áreas revegetadas também dependerá da quantidade, da qualidade e da distância dos trechos de vegetação circunvizinhos e da chegada de sementes alóctones (HARPER, 1977; GORCHOV et al., 1993; GUEVARA e LABORDE, 1993).

Considerando a transferência de propágulos como importante ferramenta para o aumento da riqueza de espécies em áreas revegetadas, tendo em vista a escassez de trabalhos sobre o tema. Este trabalho, fruto da parceria entre o Instituto de Botânica de São Paulo e a Companhia International Paper do Brasil, buscou atender a essa demanda ao fornecer subsídios para a avaliação do potencial de mata ciliar remanescente no fornecimento de propágulos para o enriquecimento de áreas revegetadas, localizadas às margens ao rio Mogi-Guaçu, SP. Para tanto, foram utilizadas informações sobre composição florística, classificação sucessional e de síndrome de dispersão de espécies arbóreas e arbustivas desse fragmento.

\section{MATERIAL E MÉTODOS}

\section{1. Área de Estudo}

A área de estudo corresponde a fragmento de vegetação ciliar remanescente, designado "mata-dosmacacos". Localiza-se em propriedade da Companhia International Paper Ltda., no Município de Conchal, nas coordenadas E:282727/N:7532654, numa altitude de $578 \mathrm{~m}$. O clima regional, segundo o sistema de Köppen (1948), é do tipo Cwa, temperado quente com estiagem no inverno. As temperaturas médias, entre os meses de janeiro e dezembro de 2000 , variaram entre $25,3^{\circ} \mathrm{C}$, no mês mais quente e $17^{\circ} \mathrm{C}$, no mês mais frio. A média de precipitação pluviométrica, no ano de 2000, na região foi em torno de $1.428 \mathrm{~mm}$, havendo déficit hídrico entre abril e junho (AQUINO, 2006).

O fragmento possui área equivalente a 89 ha e está instalado à margem direita do rio Mogi-Guaçu, em terreno plano sujeito a alagamento nos períodos chuvosos. Sua vegetação é caracterizada como mata mesófila semidecídua ribeirinha com influência fluvial sazonal (RODRIGUES, 2000) e encontra-se em estágio médio de regeneração (Resolução CONAMA n ${ }^{\circ} 1$, de 31 de janeiro de 1994), sendo protegida desde a década 
de 1960. O entorno imediato, durante o levantamento florístico, foi representado por plantio de laranja e campo antrópico. A matriz ecológica original corresponde à vegetação semidecídua.

\subsection{Caracterização Sucessional e da Síndrome de Dispersão das Espécies Arbóreas e Arbustivas}

A caracterização sucessional e da síndrome de dispersão das espécies arbóreas e arbustivas presentes na "Mata-dos-Macacos" foi efetuada a partir de levantamento florístico realizado pela empresa, entre os meses de março e novembro de 2000, em parceria com a Universidade Estadual de Campinas (UNICAMP). O material correspondente ao levantamento florístico encontra-se depositado no Herbário da UNICAMP (UEC). As espécies foram classificadas segundo o sistema de Cronquist (1981).

A classificação sucessional das espécies inventariadas seguiu os critérios estabelecidos por Budowski (1965), abrangendo os seguintes grupos ecológicos: pioneiras $(\mathrm{Pi})$; secundárias iniciais $(\mathrm{Si})$; secundárias tardias $(\mathrm{St})$ e de espécies clímax $(\mathrm{Cl})$, havendo o acréscimo de categoria referente às espécies típicas de sub-bosque ( $\mathrm{Sb}$ ), segundo proposta de Tabarelli et al. (1993). A caracterização da síndrome de dispersão das espécies seguiu os critérios propostos por Pij1 (1972), sendo empregadas as seguintes categorias: autocoria (Auto), anemocoria (Anemo), zoocoria (Zoo) e demais síndromes (Outro). As informações apresentadas tiveram como referência os trabalhos de Carmo e Morellato (2000), Rodrigues e Nave (2000), Silva et al. (2004) e Pinto et al. (2005), bem como o Anexo da SMA 21, de 21/11/2001.

A partir dos resultados foram realizados cálculos percentuais das classes sucessionais e das síndromes de dispersão registradas. Também foi aplicado o índice de similaridade de Sørensen (MAGURRAN, 1988) para comparação florística entre a "Mata-dosMacacos" e inventários realizados na região de MogiGuaçu e entorno: (a) em trechos de vegetação ciliar na Reserva Biológica de Mogi-Guaçu, Martinho Prado (MANTOVANI et al., 1989); (b) em vegetação semidecídua remanescente, Horto Mogi-Guaçu, MogiGuaçu (dados fornecidos pela empresa), (c) em vegetação semidecídua correspondente à Reserva Municipal de Santa Genebra, Campinas (GROMBONEGUARATINI e RODRIGUES, 2002).

\section{RESULTADOS E DISCUSSÕES}

Durante a realização deste trabalho, a "Mata-dosMacacos" foi representada por mosaico florístico e fisionômico, com predomínio de estratificação bem definida. A vegetação apresentou dossel predominantemente contínuo, com indivíduos arbóreos medindo entre $8 \mathrm{~m}$ e $12 \mathrm{~m}$ de altura, com grande número de indivíduos das famílias Anacardiaceae, Caesalpinaceae, Euphorbiaceae, Fabaceae, Meliaceae e Mimosaceae. Houve presença de indivíduos emergentes de até $18 \mathrm{~m}$ de altura, aproximadamente, distribuídos por todo o fragmento e representados principalmente pelas famílias Apocynaceae e Lecytidaceae. No sub-bosque foi registrada a predominância, em número de espécies e de indivíduos, das famílias Melastomataceae, Rubiaceae e Myrtaceae. Também foi observado considerável número de espécies herbáceas e de lianas que, no entanto, não foram registradas pelo levantamento florístico. Dentre as lianas, foram observadas as famílias Asteraceae, Bignoniaceae, Cactaceae, Convolvulaceae e Sapindaceae.

No levantamento florístico foram registradas 99 espécies arbóreas e arbustivas, pertencentes a 38 famílias (Tabela 1). As famílias com maior número de espécies foram Euphorbiaceae, Fabaceae, Myrtaceae e Rubiaceae, destacando-se que a riqueza registrada para a família Euphorbiaceae encontra paralelo em levantamentos realizados em matas ciliares no Estado de São Paulo (BERTONI e MARTINS, 1987; ROZZAe RIBEIRO, 1992; SILVA e SOARES, 2003; SALIS et al., 1994).

Mantovani e colaboradores (1989) identificaram em trechos de vegetação ciliar na Reserva Biológica de Mogi-Guaçu, em Martinho Prado, 139 espécies, distribuídas em 48 famílias; a riqueza florística foi variável e esteve associada à penetração de luz e fertilidade do solo. Gibbs e Leitão-Filho (1978) identificaram para a Mata-da-Figueira, localizada às margens do rio MogiGuaçu, também em Martinho Prado, 59 espécies arbóreas e arbustivas, com predomínio em número de indivíduos de Sebastiania klotzschiana e Cyclolobium vecchii.

Grombone-Guaratini e Rodrigues (2002), na Fazenda Santa Genebra, Campinas, registraram um mosaico vegetacional, identificando um total de 153 espécies de indivíduos arbustivos e arbóreos, além de 33 espécies de lianas e 14 espécies herbáceas. No Horto Mogi-Guaçu foram amostradas 105 espécies e 42 famílias; as espécies com maior número de indivíduos foram: Croton urucurana, Croton floribundus, Cecropia pachystachya, Tapirira guianensis, Casearia sylvestris e Luehea paniculata.

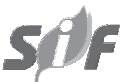

R. Árvore, Viçosa-MG, v.33, n.2, p.349-358, 2009 
Tabela 1 - Listagem das famílias e espécies arbóreas e arbustivas registradas em levantamento florístico realizado, no ano de 2000, na "mata-dos-macacos", em Conchal, SP, com os respectivos grupos sucessionais (GS), síndromes de dispersão (SD) e dados de literatura: Mantovani et al. (1999) (M); Report Ipaper (H); Grombone-Guaratini e Rodrigues(2002) (G)

Table 1 - List of the tree and scrub species sampled in floristic surveys conducted in 2000, at the "Mata-dos-macacos", in Conchal, SP, Brazil, followed by their respective successional groups $(\boldsymbol{S G})$, dispersal syndromes (DS) and literature data: Mantovani et al. (1999) (M); Report Ipaper (H); Grombone-Guaratini and Rodrigues(2002)(G)

\begin{tabular}{|c|c|c|c|}
\hline Família/ Espécie/Autor & Nome popular & GS / SD & $\mathbf{M} / \mathbf{H} / \mathbf{G}$ \\
\hline \multicolumn{4}{|l|}{ Anacardiaceae } \\
\hline Astronium graveolens Jacq. & Guaritá & St/Anemo & M \\
\hline Tapirira guianensis Aublet. & Peito-de-pombo & $\mathrm{Pi} / \mathrm{Zoo}$ & $\mathrm{H}-\mathrm{M}$ \\
\hline \multicolumn{4}{|l|}{ Annonaceae } \\
\hline Duguetia lanceolata A. St. - Hil. & Pindaíba & $\mathrm{Si} / \mathrm{Zoo}$ & G-M \\
\hline Rollinia emarginata Schltdl. & - & $-/$ Zoo & - \\
\hline \multicolumn{4}{|l|}{ Apocynaceae } \\
\hline Aspidosperma polyneuron Müll Arg. & Peroba-rosa & $\mathrm{Cl}$ /Anemo & G \\
\hline Aspidosperma ramiflorum Müll Arg. & Guatambu & St/Anemo & - \\
\hline \multicolumn{4}{|l|}{ Arecaceae } \\
\hline Euterpe edulis Mart. & Jjussara & St/Zoo & \\
\hline Syagrus romanzoffiana (Cham.) Glassman & Jerivá & $\mathrm{Si} / \mathrm{Zoo}$ & $\mathrm{H}-\mathrm{G}$ \\
\hline \multicolumn{4}{|l|}{ Bignoniaceae } \\
\hline Tabebuia chrysotricha (Mart. ex DC.) Standl. & Ipê-amarelo & St/Anemo & - \\
\hline Tabebuia umbellata (Sond.) Sandwith & Ipê-amarelo-do-brejo & St/Anemo & $\mathrm{H}$ \\
\hline Tabebuia sp. & - roxo & - /Anemo & $\mathrm{H}$ \\
\hline \multicolumn{4}{|l|}{ Bombacaceae } \\
\hline \multicolumn{4}{|l|}{ Boraginaceae } \\
\hline Cordia superba Cham. & Babosa-branca & $\mathrm{Si} / \mathrm{Zoo}$ & - \\
\hline Patagonula americana $\mathrm{L}$. & Guaiuvira & $\mathrm{Si} / \mathrm{Zoo}$ & - \\
\hline \multicolumn{4}{|l|}{ Caesalpinoidaceae } \\
\hline Copaifera langsdorffii Desf. & Copaíba & $\mathrm{St} / \mathrm{Zoo}$ & H-G-M \\
\hline Hymenaea courbaril $\mathrm{L}$. & Jatobá & St/Zoo & M \\
\hline \multicolumn{4}{|l|}{ Cecropiaceae } \\
\hline Cecropia pachystachya Trec. & Embaúba & $\mathrm{Pi} / \mathrm{Zoo}$ & H-G \\
\hline \multicolumn{4}{|l|}{ Celastraceae } \\
\hline Maytenus sp. & - & $\begin{array}{l}\mathrm{St} / \mathrm{ZOO} \\
\mathrm{Sb} /-\end{array}$ & $\overline{\mathrm{H}-\mathrm{G}}$ \\
\hline \multicolumn{4}{|l|}{ Clusiaceae } \\
\hline Calophyllum brasiliense Cambess. & |Mangue & St/Zoo & M \\
\hline \multicolumn{4}{|l|}{ Combretaceae } \\
\hline \multicolumn{4}{|l|}{ Ebenaceae } \\
\hline \multicolumn{4}{|l|}{ Euphorbiaceae } \\
\hline Actinostemon communis Pax. & - & -/Auto & - \\
\hline Alchornea glandulosa Poepp.\& Endl. & Tapiá & $\mathrm{Pi} / \mathrm{Zoo}$ & $\mathrm{H}$ \\
\hline Croton floribundus Spreng. & Capixingui & $\mathrm{Pi} /$ Auto & H-G-M \\
\hline Croton urucurana Baill. & Sangra-d'água & $\mathrm{Pi} /$ Auto & H-M \\
\hline Margaritaria sp. & - & St / Auto & - \\
\hline Savia dictyocarpa Müll. Arg. & - & $\mathrm{Cl} /$ Auto & - \\
\hline Sebastiania edwalliana Pax \& Hoffm. & Sebastiania & —/Auto & $\mathrm{H}-\mathrm{G}$ \\
\hline Sebastiania klotzchiana (Müll Arg.) Müll Arg. & Sebastiania & $\mathrm{Pi} /$ Auto & M \\
\hline Sebastiania serrata (Baill.) Müll Arg. & - & Si/Auto & M \\
\hline
\end{tabular}

R. Árvore, Viçosa-MG, v.33, n.2, p.349-358, 2009 
Tabela 1 - Cont.

Table 1 -Cont.

\begin{tabular}{|c|c|c|c|}
\hline Família/ Espécie/Autor & Nome popular & GS / SD & $\mathbf{M} / \mathbf{H} / \mathbf{G}$ \\
\hline \multicolumn{4}{|l|}{ Fabaceae } \\
\hline Cyclolobium vecchii A. Samp. ex Hoehne & Llouveira & St/Outro & - \\
\hline Dalbergia frutescens (Vell.) Britton & Caroba-brava & Si/Anemo & - \\
\hline Lonchocarpus guillemianus (Tu1.) Malme & Embira-de-sapo & Si/Anemo & - \\
\hline Machaerium brasiliense Vog. & Jacarandá & St/Anemo & $\mathrm{H}-\mathrm{G}$ \\
\hline Machaerium aculeatum Raddi. & Bico-de-pato & $\mathrm{Pi} /$ Anemo & $\mathrm{H}$ \\
\hline Myroloxylum peruiferum L. f. & - & St/Anemo & G \\
\hline \multicolumn{4}{|l|}{ Flacourtiaceae } \\
\hline Casearia gossypiosperma Briquet & - & $\mathrm{Sb} / \mathrm{Anemo}$ & H-G-M \\
\hline Casearia sylvestris $\mathrm{Sw}$. & Erva-de-lagarto & $\mathrm{Pi} / \mathrm{Zoo}$ & G-M \\
\hline \multicolumn{4}{|l|}{ Lauraceae } \\
\hline Endicheria paniculata (Speng.) Macbr. & Canela-de-frade & St/Zoo & H-M \\
\hline Nectandra megapotamica (Spreng.) Mez & Canelinha & $\mathrm{Si} / \mathrm{Zoo}$ & H-G-M \\
\hline Nectandra rigida (H. B. K.) Ness. & Canela-ferrugem & $\mathrm{Si} / \mathrm{Zoo}$ & $\mathrm{H}$ \\
\hline \multicolumn{4}{|l|}{ Lecytidaceae } \\
\hline Cariniana estrellensis (Raddi) Kuntze & Jequitibá-branco & St/Anemo & G-M \\
\hline Cariniana legalis (Mart.) Kuntze & Jequitibá & St/Anemo & G \\
\hline \multicolumn{4}{|l|}{ Melastomataceae } \\
\hline Miconia sp. & - & $\mathrm{Sb} / \mathrm{Zoo}$ & \\
\hline Leandra sp. & - & $\mathrm{Sb} /-$ & \\
\hline \multicolumn{4}{|l|}{ Meliaceae } \\
\hline Guarea macrophylla Vahl & Marinheiro & $\mathrm{Sb} / \mathrm{Zoo}$ & M \\
\hline Trichilia casaretti C. DC. & - & -/Zoo & - \\
\hline Trichilia catigua A. Juss. & - & $\mathrm{Sb} / \mathrm{Zoo}$ & G-M \\
\hline Trichilia pallida $\mathrm{Sw}$ & Baga-de-morcego & St/Zoo & H-G-M \\
\hline \multicolumn{4}{|l|}{ Mimosaceae } \\
\hline Albizia niopoides (Spruce ex Benth.) Burkart & Angico-branco & St/Outro & - \\
\hline Inga luschnatiana Benth. & - & —/Zoo & $\mathrm{H}-\mathrm{G}$ \\
\hline Inga marginata $\mathrm{Will}$ & Ingá-feijão & $\mathrm{Pi} / \mathrm{Zoo}$ & $\mathrm{H}-\mathrm{G}$ \\
\hline Inga uruguensis Hook \& Arn. & Ingá-do-brejo & $\mathrm{Si} / \mathrm{Zoo}$ & G \\
\hline Piptadenia gonoacantha (Mart.) J. F. Macbr. & Pau-jacaré & Si/Outro & - \\
\hline \multicolumn{4}{|l|}{ Monimiaceae } \\
\hline Mollinedia argyrogina Perkins & - & $-/$ Zoo & M \\
\hline Siparuna guianensis Aubl. & - & $\mathrm{Pi} / \mathrm{Zoo}$ & $\mathrm{H}$ \\
\hline Siparuna tomentosa Perkins & - & -/Zoo & - \\
\hline \multicolumn{4}{|l|}{ Moraceae } \\
\hline Brosimum sp. & Grão-de-galo & -/Zoo & H-G-M \\
\hline Fícus guaranitica Chodat & Figueira-branca & St/Zoo & $\mathrm{H}$ \\
\hline \multicolumn{4}{|l|}{ Myrsinaceae } \\
\hline Rapanea ferruginea (Ruiz \& Pav.) Mez & - & $\mathrm{Pi} / \mathrm{Zoo}$ & $\mathrm{H}$ \\
\hline Rapanea guianensis Aubl. & Capororoca & $\mathrm{Pi} / \mathrm{Zoo}$ & $\mathrm{H}$ \\
\hline \multicolumn{4}{|l|}{ Myrtaceae } \\
\hline Calycorectes acutatus (Miq.) Toledo & Araçá-da- serra & $\mathrm{Sb} / \mathrm{Zoo}$ & - \\
\hline Calyptranthes concinna DC. & Guamirim-de-faxo & $\mathrm{Sb} / \mathrm{Zoo}$ & $\mathrm{G}$ \\
\hline Campomanesia guazumifolia (Cambess.) O. Berg & Sete-capotes & St/Zoo & M \\
\hline Campomanesia xanthocarpa $\mathrm{O}$. Berg & Guabiroba & St/Zoo & - \\
\hline Eugenia aurata $\mathrm{O}$. Berg & Araçá & $\mathrm{Sb} / \mathrm{Zoo}$ & - \\
\hline Eugenia glazioviana Kiaersk. & Guamirim & $\mathrm{Sb} / \mathrm{Zoo}$ & - \\
\hline Eugenia involucrata DC. & Cerejeira & $\mathrm{Sb} / \mathrm{Zoo}$ & $\mathrm{H}$ \\
\hline Eugenia ligustrina Willd. & - & $\mathrm{Sb} / \mathrm{Zoo}$ & G \\
\hline Eugenia uniflora $\mathrm{L}$. & Pitanga & $\mathrm{Sb} / \mathrm{Zoo}$ & - \\
\hline
\end{tabular}


Tabela 1 - Cont.

Table 1 - Cont

\begin{tabular}{|c|c|c|c|}
\hline Família/ Espécie/Autor & Nome popular & GS / SD & $\mathbf{M} / \mathbf{H} / \mathbf{G}$ \\
\hline \multicolumn{4}{|l|}{ Myrtaceae } \\
\hline Myrcia rostrata DC. & - & $\mathrm{Sb} / \mathrm{Zoo}$ & $\mathrm{H}-\mathrm{G}$ \\
\hline Myrciaria tenella $\mathrm{O}$. Berg & Cambuí & $\mathrm{Sb} / \mathrm{Zoo}$ & - \\
\hline Myrtaceae 1 & - & $\mathrm{Sb} / \mathrm{Zoo}$ & - \\
\hline Myrtaceae 2 & - & $\mathrm{Sb} / \mathrm{Zoo}$ & - \\
\hline Myrtaceae 3 & - & $\mathrm{Sb} / \mathrm{Zoo}$ & - \\
\hline \multicolumn{4}{|l|}{ Nyctaginaceae } \\
\hline Guapira sp. & - & St/Zoo & \\
\hline \multicolumn{4}{|l|}{ Phytholacaceae } \\
\hline Gallesia integrifolia (Spreng.) Harms & Pau-d'alho & St/Zoo & \\
\hline \multicolumn{4}{|l|}{ Polygonaceae } \\
\hline Ruprechtia sp. & - & St/Anemo & \\
\hline \multicolumn{4}{|l|}{ Proteaceae } \\
\hline Roupala montana Aubl. & - & $\mathrm{Si} /$ Anemo & $\mathrm{H}$ \\
\hline \multicolumn{4}{|l|}{ Rubiaceae } \\
\hline Coffea arabica $\mathrm{L}$. & Café & Ex/Zoo & G \\
\hline Genipa americana $\mathrm{L}$. & Genipapo & St/Zoo & M \\
\hline Ixora sp. & - & $\mathrm{Sb} / \mathrm{Zoo}$ & H-G-M \\
\hline Psychotria nemorosa Gardner & - & $\mathrm{Sb} / \mathrm{Zoo}$ & - \\
\hline Psychotria vellosiana Benth. & - & $\mathrm{Sb} / \mathrm{Zoo}$ & M \\
\hline Psychotria sp. & - & $\mathrm{Sb} / \mathrm{Zoo}$ & H-G-M \\
\hline \multicolumn{4}{|l|}{ Rutaceae } \\
\hline Esenbeckia sp. & - & -1- & H-G \\
\hline Zanthoxylum rhoifolium Lam. & Mamica-de-cadela & $\mathrm{Si} / \mathrm{Zoo}$ & H-G-M \\
\hline Zanthoxylum riedelianum Lam. & Mamica-de-porca & $\mathrm{Pi} / \mathrm{Zoo}$ & H-G-M \\
\hline \multicolumn{4}{|l|}{ Sapindaceae } \\
\hline Cupania vernalis Cambess & Camboatã & $\mathrm{Si} / \mathrm{Zoo}$ & G-M \\
\hline Matayba elaegnoides Radlk. & Camboatã-branco & St/Zoo & $\mathrm{H}-\mathrm{M}$ \\
\hline \multicolumn{4}{|l|}{ Sapotaceae } \\
\hline Chrysophyllum gonocarpum (Mart.\& Eich1.) Engl. & Pau-de-leite & St/Zoo & G \\
\hline Pouteria sp. & - & $\mathrm{Si} / \mathrm{Zoo}$ & H-G-M \\
\hline Sapotaceae sp 1 & - & $-1-$ & - \\
\hline \multicolumn{4}{|l|}{ Simaroubaceae } \\
\hline Picramia sellowiana Planch. & - & —/Zoo & - \\
\hline \multicolumn{4}{|l|}{ Solanaceae } \\
\hline Solanaceae sp1 & - & $\mathrm{Sb} / \mathrm{Zoo}$ & - \\
\hline \multicolumn{4}{|l|}{ Symplocaceae } \\
\hline Symplocus sp. & - & —/Zoo & M \\
\hline \multicolumn{4}{|l|}{ Tiliaceae } \\
\hline Luehea paniculata Mart. & Açoita-cavalo & $\mathrm{Si} /$ Anemo & $\mathrm{H}$ \\
\hline \multicolumn{4}{|l|}{ Vochysiaceae } \\
\hline Qualea multiflora Mart. & - & $\mathrm{Si} /$ Anemo & M \\
\hline
\end{tabular}

Total: 38 famílias e 99 espécies

$\mathrm{Pi}=$ pioneira $; \mathrm{Si}=$ secundária inicial $; \mathrm{St}=$ secundária tardia $; \mathrm{Cl}=$ clímax $; \mathrm{Sb}=$ sub-bosque $;$ Ex = exótica $; \mathrm{Zoo}=$ zoocoria $;$ Anemo = anemocoria; Auto = autocoria; Outro = barocoria, hidrocoria...; e Nid. = não identificada.

$\mathbf{P i}=$ pioneer; $\mathbf{S i}=$ initial secondary $; \mathbf{S t}=$ late secondary $; \mathbf{C l}=$ climax $; \mathbf{S b}=$ understore $; \mathbf{E x}=$ exotic $; \mathbf{Z o o}=$ zoochory $; \mathbf{A n e m o}=$ anemochory; Auto = autochory; Other = barochory, hidrochory; and Nid. = not identified

Os valores registrados para número de espécies e de famílias na "Mata-dos-Macacos" foram intermediários aos obtidos nas matas ciliares da região e inferiores aos registrados nos fragmentos de vegetação semidecídua. Entre as espécies amostradas, somente seis estiveram presentes nos três trabalhos utilizados como referência (GROMBONE-GUARATINI e RODRIGUES, 2002; MANTOVANI et al., 1989; Relatório interno Ipaper), sendo elas: Copaifera langsdorffii, Croton floribundus, Trichilia pallida, Psychotria

R. Árvore, Viçosa-MG, v.33, n.2, p.349-358, 2009 
nemorosa, Zanthoxylum rhoifolium e Zanthoxylum riedelianum.

O índice de similaridade de Sørensen apontou maior proximidade florística entre a "Mata-dos-Macacos" e o Horto Mogi-Guaçu (35,30\%); a similaridade foi de 26,90\% com relação à vegetação ciliar da Reserva Biológica de Mogi-Guaçu e de 26,20\% com a Santa Genebra. Características distintas entre fragmentos ciliares próximos se devem, entre outros fatores, ao histórico de antropização e fragmentação, bem como condições bióticas e abióticas específicas de cada um deles (RODRIGUES e NAVE, 2000).

Leitão-Filho e colaboradores (2003) realizaram inventário e caracterização de fragmentos de vegetação florestal remanescente nas Bacias dos Rios Piracicaba e Capivari. Nos fragmentos localizados em áreas de ocorrência de Floresta Estacional Semidecidual foram registradas 569 espécies, distribuídas em 75 famílias, desse total, 16 espécies foram registradas entre $70 \%$ e $100 \%$ das localidades, sendo consideradas espécies muito frequentes: Astronium graveolens, Aspidosperma polyneuron, Syagrus romanzofianum, Bauhinia forficata, Copaifera langsdorffii, Croton fioribundus, Myroxylon peruiferum, Casearia sylvestris, Trichilia catigua, Zanthoxylon rhoifolium, também registradas no levantamento realizado na "Mata-dos-Macacos", Cedrela fissilis, Cabralea canjerana, Trichilia claussenii, Esenbeckia febrifuga, Centrolobium tomentosum, Holocalyx balansae e Machaerium stipitatum.

Em Mogi-Guaçu, Gibbs e colaboradores (1980) amostraram como espécies mais importantes na vegetação ciliar: Sebastiania klotzschiana, Cyclolobium vecchii, Genipa americana, também registradas na "Mata-dos-Macacos", Inga vera, Alchornea triplinervia e Guarea trichilioides. Lobo e Joly (2000) citaram entre as espécies tolerantes a alagamento Sebastiana klotzschiana como espécie típica de diques; Hymenaea courbaril var. stilbocarpa e Genipa americana como características de depressões; Chorisia speciosa como frequente em áreas de inundações esporádicas e Copaifera langsdorffii de ampla distribuição.

Quanto às classes sucessionais registradas na "Mata-dos-Macacos", foi verificado um equilíbrio na quantidade de espécies secundárias tardias (28 espécies, $28,3 \%$ do total) e aquelas típicas de sub-bosque (23 espécies, 23,2\% do total), destacando-se em número de espécies as famílias Bignoniaceae, Caesalpinoidaceae, Fabaceae, Lecytidaceae, Myrtaceae e Rubiaceae. Os demais grupos foram representados por espécies secundárias iniciais $(18,9 \%)$, destacando-se em Boraginaceae e Lauraceae; pioneiras (13,1\%), destacando-se em Euphorbiaceae; espécies clímax (3,0\%), presentes nas famílias Apocynaceae, Combretaceae e Euphorbiaceae; e exóticas $(1,0 \%)$ e espécies que não tiveram classificação sucessional identificada $(13,1 \%)$ (Figura 1a e Tabela 1).
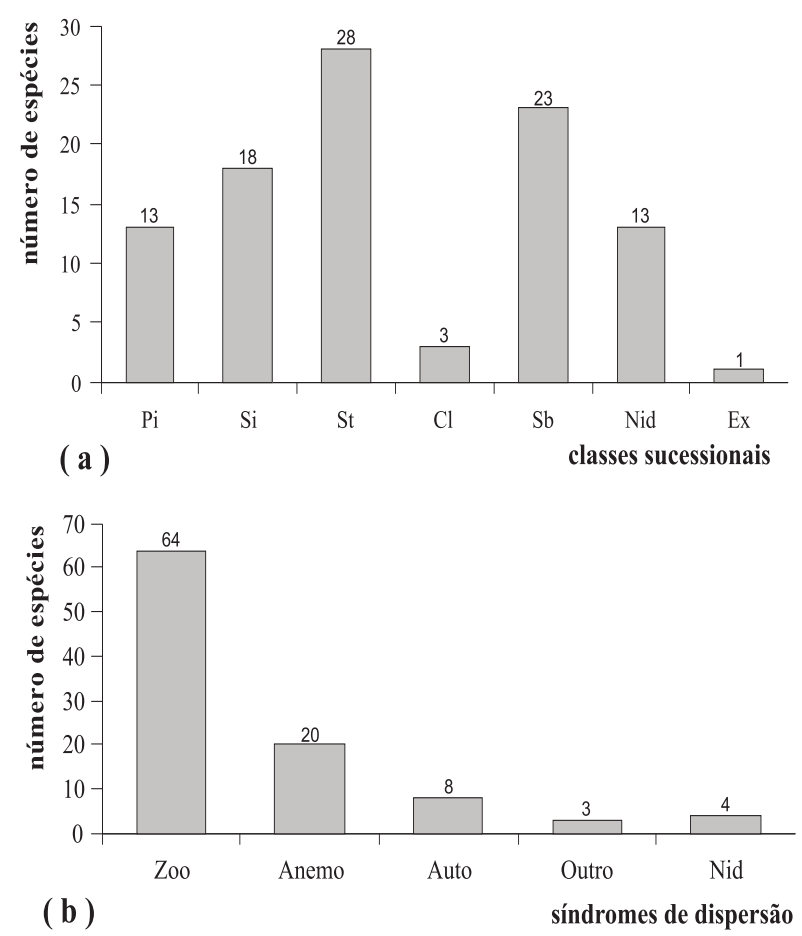

Figura 1 - Número de espécies arbóreas e arbustivas por grupo sucessional (a) e síndrome de dispersão (b) registrados em levantamento florístico realizado, no ano de 2000, na "mata-dos-macacos", Município de Conchal, São Paulo, Brasil. $\mathrm{Pi}=$ pioneira; $\mathrm{Si}$ $=$ secundária inicial $; \mathrm{St}=$ secundária tardia $; \mathrm{Cl}=$ clímax; Sb = sub-bosque; Ex = exótica; Zoo = zoocoria; Anemo = anemocoria; Auto = autocoria; Outro $=$ barocoria, hidrocoria...; e Nid. = não identificada.

Figure 1-Number of species per successional group (a) and dispersal syndromes $(b)$ recorded in floristic surveys conducted in 2000, at the "Mata-dos-macacos", in Conchal, $\mathrm{SP}$, Brazil. $\mathbf{P i}=$ pioneer; $\mathbf{S i}=$ initial secondary; $\boldsymbol{S t}=$ late secondary; $\boldsymbol{C l}=$ climax $; \boldsymbol{S b}$ $=$ understore $; \boldsymbol{E x}=$ exotic $; \boldsymbol{Z o o}=$ zoochory; Anemo = anemochory; Auto $=$ autochory; Other $=$ barochory, hidrochory; and Nid. $=$ not identified

R. Árvore, Viçosa-MG, v.33, n.2, p.349-358, 2009 
A síndrome de dispersão predominante entre as espécies arbóreas e arbustivas foi a zoocoria, identificada em 64 espécies, 64,6\% do total amostrado. Essa síndrome esteve fortemente associada às espécies de sub-bosque, mas também foi representativa nas pioneiras, secundárias iniciais e secundárias tardias, destacando-se, em número de espécies, as famílias Myrtaceae, Rubiaceae e Meliaceae. A anemocoria representou 20,2\% dos casos, destacando-se nas famílias Bignoniaceae e Fabaceae. A autocoria, registrada para $8,1 \%$ das espécies, foi predominante em Euphorbiaceae. Outras formas de dispersão e não identificadas apresentaram mesma proporção, com 4,0\% dos casos (Figura 1b e Tabela 1).

Valores bastante próximos aos obtidos na "Matados-Macacos" foram registrados em estudo realizado na bacia do rio Tibagi, onde Carmo e Morellato (2000) observaram que, entre 261 espécies arbóreas e arbustivas amostradas, $67,8 \%$ foram zoocóricas, $15,7 \%$ anemocóricas, $7,7 \%$ apresentaram outras formas de dispersão e 8,8\% não tiveram síndrome de dispersão identificada. De fato, estudos demonstram que a zoocoria é o mecanismo de dispersão mais importante em florestas tropicais (GENTRY, 1982; STILES, 1989). Segundo Vieira e colaboradores (2002), a propagação de sementes por anemocoria apresenta maior eficiência em áreas mais abertas, justificando menor representatividade dessa síndrome em florestas úmidas.

\section{CONCLUSÕES}

O principal interesse deste trabalho foi a verificação de qualidades associadas ao fragmento de estudo que pudessem contribuir para o aumento de diversidade em áreas revegetadas, existentes na região de MogiGuaçu, tendo em vista a manutenção de características da vegetação original da região. Os resultados; como: (a) o expressivo número de espécies arbóreas e arbustivas, muitas das quais também registradas em fragmentos ciliares e semideciduais e (b) o elevado número de espécies secundárias e típicas de sub-bosque, com predomínio de espécies zoocóricas indicou a viabilidade da utilização da "Mata-dos-Macacos" como fonte de propágulos para atividades de revegetação e, ou, manejo na região de Mogi-Guaçu, em áreas ciliares revegetadas que apresentem baixa resiliência e a dispersão de diásporos se mostre dificultada. Destaca-se, no entanto, que a dinâmica sucessional ou de regeneração em uma área dependerá de sua interação com o entorno imediato, em função da quantidade, da qualidade e da distância dos trechos de vegetação remanescente circunvizinhos, bem como da presença de agentes dispersores. Qualquer prática de repovoamento vegetal deve considerar, portanto, além da alta diversidade de espécies e de formas de vida, a necessidade de agentes polinizadores e dispersores, além de contemplar grupos sucessionais e formas de vida variadas que apresentem dispersão dificultada até ambientes mais isolados.

\section{AGRADECIMENTOS}

Ao Instituto de Botânica de São Paulo e à International Paper do Brasil, pelo auxílio e viabilização deste trabalho.

\section{REFERÊNCIAS}

AQUINO, C. Avaliação de três formas de enriquecimento em área ciliar revegetada junto ao rio Mogi-Guaçu, SP. 2006. 154f. Dissertação (Mestrado em Biologia Vegetal) - Universidade Estadual Júlio de Mesquita-Filho, Rio Claro, 2006.

BARBOSA, L. M. (Coord.) SIMPÓSIO SOBRE MATA CILIAR, 1., 1989, Campinas. Anais... Campinas: Fundação Cargill, 1989.

BARBOSA, L. M. (Coord.). SIMPÓSIO SOBRE RECUPERAÇÃO DE ÁREAS DEGRADADAS (RAD) COM ÊNFASE EM MATAS CILIARES E WORKSHOP SOBRE RECUPERAÇÃO DE ÁREAS DEGRADADAS NO ESTADO DE SÃO PAULO: AVALIAÇÃO DA APLICAÇÃO E APRIMORAMENTO DA RESOLUÇÃO SMA 47/ 03. 2006, São Paulo. Anais... São Paulo: Secretaria do Meio Ambiente, Instituto de Botânica de São Paulo, 2006.

BARBOSA, L. M. et al. Estudos sobre o estabelecimento e desenvolvimento de espécies com ampla ocorrência em mata ciliar. Revista do Instituto Florestal, v.4, p.605-608, 1992.

BARBOSA, L. M. et al. Recuperação florestal com espécies nativas no Estado de São Paulo: Pesquisas apontam mudanças necessárias.

Florestar Estatístico, v.6, n.14, p.28-34, 2003.

BAWA, K.; KRUGMAN, S. L. Reprodutive biology and genetics of tropical forest trees. In: HADLEY, M. (Ed.) Rain forest regeneration and management. Guri: 1986. p.22-28. 
BENÍTEZ-MALVIDO, J.; MARTÍNEZ-RAMOS, M. Influence of edge exposure on tree seedling species recruitment in tropical rain forest fragments. Biotropica, v.35, n.4, p.530-541, 2002.

BERTONI, J. E. A.; MARTINS, F. R. Composição florística de uma floresta ripária na Reserva Estadual de Porto Ferreira, SP. Acta Botanica Brasilica, v.1, n.1, p.17-26, 1987.

BUDOWSKY, G. Distribuition of Tropical American Rain Forest species in the light of sucession process. Turrialba, v.15, n.1, p.40-42, 1965.

CARMO, M. R. B.; MORELLATO, L. C. P. Fenologia de árvores e arbustos das matas ciliares da bacia do rio Tibagi, Estado do Paraná, Brasil. In: RODRIGUES, R. R.; LEITÃO FILHO, H. F. (Eds.) Matas Ciliares: conservação e recuperação. São Paulo: EDUSP, FAPESP, 2000. p.125-142.

CATHARINO, E.L.M. Florística de matas ciliares. In: SIMPÓSIO SOBRE MATA CILIAR, 1., 1989, Campinas. Anais... Campinas: Fundação Cargill, 1989. p.61-69.

CRONQUIST, A. The evolution and classification of flowering plants. New York: The New York Botanical Garden, 1981. 555p.

DENSLOW, J. S. Functional group diversity and responses to disturbance. In: ORIANS, G. H.; DIRZO, R.; CUSHMAN, J. H. (Eds.)

Biodiversity and ecosystem processes in tropical forests. ecological studies.. Berlin: Springer-Verlag, 1996. v.122. p.127-151.

GENTRY, A. H. Patterns of neotropical plante species diversity. Evolutionary Biology, v.15, n.1, p.1-84, 1982.

GIBBS, P. E.; LEITÃO FILHO, H. F. Floristic composition of an area of gallery forest near Mogi-Guaçu, State of São Paulo. S.E. Brazil. Revista Brasileira de Botânica, v.1, n.2, p.151-156, 1978.

GIBBS, P. E.; LEITÃO-FILHO, H. F.; ABBOT, R. J. Application of the point-centred quarter method in a floristic survey of an Gallery Forest in MojiGuaçu, SP, Brasil. Revista Brasileira de Botânica, v.3, n.1, p.17-22, 1980.
GORCHOV, D. L. et al. The role of seed dispersal in the natural regeneration of rain forest after strip-cutting in the Peruvian Amazon. Vegetatio, v.107/108, p.339-349, 1993.

GRIME, J. P. Benefits of plant diversity to ecosystems: immediate, filter and founder effects. Journal of Ecology, v.86, n.6, p.902-910, 1998.

GROMBONE-GUARATINI, M. T.; RODRIGUES, R. $R$. Seed bank and seed rain in a seasonal semideciduous forest in south-eastern Brazil. Journal of Tropical Ecology, v.18, n.5, p.759-774, 2002.

GUEVARA, S.; LABORDE, J. Monitoring seed dispersal at isolated standing trees in tropical pastures: consequences for local species availability. Vegetatio, v.107, n.1, p.319-338, 1993.

HARPER, J. L. Population biology of plants. New York: Academic Press, 1977.

KAGEYAMA, P. Y.; GANDARA, F. B. Recuperação de Áreas Ciliares. In: RODRIGUES, R. R.; LEITÃO FILHO, H. F. (Eds.) Matas Ciliares: conservação e recuperação. São Paulo: EDUSP, FAPESP, 2000. p.249-270.

KOPPEN, W. Climatologia. México: Fondo de Cultura Econômica, 1948.

LEITÃO FILHO, H. F. et al. Vegetação florestal remanescente: inventários, caracterização, manejo e recuperação nas bacias dos rios Piracicaba e Capivari. Disponível em: <http://nepam.unicamp.br/publicacoes/material/ caderno7.pdf> . Acesso em: jan de 2007.

LOBO, P. C.; JOLY, C. A. Aspectos ecofisiológicos da vegetação de mata ciliar do sudeste do Brasil. In: RODRIGUES, R. R.; LEITÃO FILHO, H. F. (Eds.) Matas Ciliares: conservação e recuperação. São Paulo: EDUSP, FAPESP, 2000. p.143-157.

LOBO, P. C.; JOLY, C. A. Ecofisiologia da germinação de sementes de Talauma ovata St Hil. (Magnoliaceae), uma espécie típica de mata de brejo. Revista Brasileira de Botânica, v.19, n.1, p.35-40, 1995.

MAGURRAN, A. E. Ecological diversity and its measurement. Princeton: Princeton University Press, 1988. 179p.

R. Árvore, Viçosa-MG, v.33, n.2, p.349-358, 2009 
MANTOVANI, W. et al. Estudo fitossociológico de áreas de mata ciliar em Mogi-Guaçu, SP, Brasil. In: SIMPÓSIO SOBRE MATA CILIAR, 1., 1989, Campinas. Anais... Campinas: Fundação Cargill, 1989. p.235-267.

METACALFE, D. J.; TURNER, L. M. Soil seed bank from lowland rain forest in Singapore: canopy-gab and litter-gap demanders. Journal of Tropical Ecology, v.14, n.1, p.103-108, 1998.

PICKETT, S. T. A.; COLLINS, S. L.; ARMESTO, J. J. A hierarchical consideration of causes and mechanisms of sucession. Vegetatio, v.69, n.1, p.109-114, 1987.

PINTO, L.V.A. et al. Estudo da vegetação como subsídios para propostas de recuperação das nascentes da bacia hidrográfica do ribeirão Santa Cruz, Lavras, MG. Revista Árvore, v.29, n.5, p.775-793, 2005.

RODRIGUES, R. R. Uma discussão nomenclatural das Formações Ciliares In: RODRIGUES, R. R.; LEITÃO FILHO, H. F. (Eds.) Matas Ciliares: conservação e recuperação. São Paulo: EDUSP, FAPESP, 2000. p.91-99.

RODRIGUES, R. R.; LEITÃO. FILHO, H. F. Matas ciliares: conservação e recuperação. São Paulo: EDUSP, FAPESP, 2000. 320p.

RODRIGUES, R. R.; NAVE, A. G. Heterogeneidade florística das matas ciliares. In: RODRIGUES, R. R.; LEITÃO FILHO, H. F. (Eds.) Matas Ciliares: conservação e recuperação. São Paulo: EDUSP, FAPESP, 2000. p.159-167.

ROZZA, A. F.; RIBEIRO, C. A. Estudo florístico e fitossociológico de fragmentos de mata ciliar dos Campos da ESALQ, Piracicaba, SP. In: CONGRESSO DA SOCIEDADE BOTÂNICA DE SÃO PAULO, 8., 1992, Campinas. Anais... São Paulo: Sociedade Botânica de São Paulo, 1992.
SALIS, S. M.; TAMASHIRO, J. Y.; JOLY, C. A. Floristica e fitossociologia do estrato arbóreo de um remanescente de mata ciliar do rio JacaréPepira, Brotas, SP. Revista Brasileira de Botânica, v.17, n.2, p.93-103, 1994.

SILVA, C. T. et al. Avaliação temporal da florística arbórea de uma floresta secundária no município de Viçosa, Minas Gerais. Revista Árvore, v.28, n.3, p.429-441, 2004.

SILVA, L. A.; SOARES, J. J. Composição florística de um fragmento de floresta estacional semidecídua no município de São Carlos-SP. Revista Árvore, v.27, n.5, p.647-656, 2003.

STILES, E. W. Fruits, seeds and dispersal agents. In: ABRAHAM, W. G. Plant - animal interactions. New York: Mc Graw Hill, 1989.

TABARELLI, M.; VILLANI, J. P.; MANTOVANI, W. Aspectos da sucessão secundária em trecho da floresta atlântica no Parque Estadual da Serra do Mar, SP. Revista do Instituto Florestal, v.5, n.1, p.101-114, 1993.

Van der PIJL, L. Principles of dispersal in higher plants. Berlim: Springer, 1972. 162p.

VÁZQUEZ-YANES, C. Notas sobre la autoecologia de los arboles pioneros de rapido crescimento de la selva tropical lluviosa. Tropical Ecology, v.21, n.1, p.103-112, 1980.

VIEIRA, D. L. M. et al. Síndrome de dispersão de espécies arbustivo-arbóreas em cerrado sensu strictu do Brasil Central e savanas amazônicas. Revista Brasileira de Botânica, v.25, n.2, p.215-220, 2002. 